\title{
SOFT SYSTEMS METHODOLOGY (SSM) NO CONTEXTO DA EDUCAÇÃO TECNOLÓGICA: CONTRIBUIÇÕES AOS PROCESSOS DE GESTÃO DO CONHECIMENTO (GC)
}

\section{SOFT SYSTEMS METHODOLOGY (SSM) IN EDUCATION TECHNOLOGY CONTEXT: CONTRIBUTIONS TO KNOWLEDGE MANAGEMENT PROCESSES (KM)}

\author{
Denize Demarche Minatti Ferreira ${ }^{1}$; Arleide Rosa da Silva ${ }^{2}$; Teresa Cristina Santos Rebello ${ }^{3}$; \\ Neri dos Santos ${ }^{4}$ \\ ${ }^{1}$ Universidade Federal de Santa Catarina - UFSC - Florianópolis - Brasil \\ dminatti@terra.com.br \\ ${ }^{2}$ Universidade Federal de Santa Catarina - UFSC - Florianópolis - Brasil \\ arosa@furb.br \\ ${ }^{3}$ Universidade Estadual de Santa Catarina - UDESC - Florianópolis - Brasil \\ tete.rebello@gmail.com \\ ${ }^{4}$ Universidade Federal de Santa Catarina - UFSC - Florianópolis - Brasil \\ neri@egc.ufsc.br
}

\begin{abstract}
Resumo
Este artigo descreve os procedimentos para a utilização da Soft Systems Methodology (SSM), bem como sua adequação ao contexto da educação tecnológica. Analisa o processo avaliativo em cursos superiores de tecnologia e como o mesmo pode contribuir para a construção do conhecimento. Propõe, para tanto, a aplicação dessa metodologia em uma Instituição de Educação Tecnológica na tentativa de solucionar dificuldades no processo avaliativo, considerando a subjetividade dos resultados baseados em conceitos, e não em notas como no sistema tradicional; o tempo existente para a integralização das disciplinas; e, o processo de aprendizagem inerente a esta modalidade, construída sobre o conhecimento teórico (saber), sobre o desenvolvimento de uma habilidade prática (saber fazer) e sobre o desenvolvimento de atitudes em esferas específicas da atividade humana (saber ser). Acredita-se que a adoção da referida metodologia em processos educacionais que envolvam o gerenciamento do conhecimento nas Instituições de Educação Tecnológica contribui para a agregação de valor aos cursos superiores de tecnologia além de minimizar a distância entre os níveis organizacionais a fim de aperfeiçoar o processo de gestão.
\end{abstract}

Palavras-chave: Soft Systems Methodology; Educação Tecnológica; Gerenciamento do Conhecimento; Cursos Superiores de Tecnologia. 


\section{Introdução}

Nenhum país pode desenvolver-se e tornar-se independente sem um sólido sistema de educação superior. Num mundo em que o conhecimento se sobressai em relação aos recursos materiais como fator de desenvolvimento humano, a importância da educação superior e de suas instituições é cada vez maior, já que nelas de fato se produz conhecimento.

Os Cursos Superiores de Tecnologia, criados por meio do inciso II, do artigo 44, da Lei 9.394/96 (LDB), apresentam-se como um alicerce para este sistema, contribuindo para o aumento da empregabilidade, na qual a postura pessoal pró-ativa e o conhecimento agregado pelo trabalhador são peças-chave para seu sucesso profissional. Tais cursos foram criados com o objetivo de graduar profissionais e inseri-los de forma mais rápida e direcionada no mercado de trabalho, já que prevêem períodos de integralização normalmente mais curtos do que os cursos tradicionais de graduação (bacharelados e licenciaturas). A formação é direcionada para aplicação, desenvolvimento e difusão de tecnologias, com foco em gestão de processos de produção de bens e serviços e capacidade empreendedora. A visão desta modalidade de ensino superior é focada em ensinar o aluno o saber ser e o fazer, e não somente um conhecimento teórico sobre determinado conteúdo.

O Ministério da Educação e do Desporto e o Ministério do Trabalho, desde 1995, segundo estudo elaborado por Lima Filho (2007) vêm desenvolvendo estudos e ações com vistas a reformar e redirecionar as instituições de ensino técnico-profissional conhecido como a "Reforma da Educação Profissional". Abrange 134 instituições federais e têm igualmente impactos sobre as redes estaduais de ensino médio e profissional, o Sistema "S" e outras instituições privadas que trabalham com a educação profissional.

Os Cursos Superiores de Tecnologia pretendem ir ao encontro das necessidades da nova educação profissional do país, que pressupõe um vínculo permanente com o mundo do trabalho e a prática social. Neste sentido, as Instituições de Educação Superior do nosso país buscam ofertar cursos sintonizados com as demandas de mercado, dos cidadãos e da sociedade, através de um ensino contextualizado, que supera a dicotomia teoria/prática, uma vez que esta prática profissional constitui e organiza o desenvolvimento curricular dos mesmos. O MEC aponta que em 2004 o número de ingressantes nos Cursos Superiores de Tecnologia representou 7,2\% do total no nível superior do País e 3,7\% dos alunos respectivamente matriculados.

Uma análise da inclusão de disciplinas voltadas à educação tecnológica na educação geral em vários países europeus como, por exemplo, a Áustria - em trabalho desenvolvido por Manfredinho (2000) - demonstra a amplitude de sua importância para despertar nas pessoas mais 
comuns uma atitude tecnológica e o desenvolvimento da capacidade de responder a problemas tecnológicos correntes.

Considerando a alta complexidade do sistema educacional tecnológico, repleto de relações e interações que se adequam ao uso da metodologia de sistemas flexíveis, propomos a adoção da metodologia SSM (Soft Systems Methodology) ou Metodologia dos Sistemas Flexíveis para solucionar dificuldades no processo avaliativo em Cursos Superiores de Tecnologia, considerando o tempo existente para a integralização das disciplinas e o processo de aprendizagem inerente à esta modalidade, construída sobre o conhecimento teórico (saber), sobre o desenvolvimento de uma habilidade prática (saber fazer) e sobre o desenvolvimento de atitudes em esferas específicas da atividade humana (saber ser).

A aplicação da SSM aborda quatro atividades principais; inicialmente procura-se entender uma situação problemática, considerando as dimensões humanas, sociais, políticas e culturais, passa-se então a formular modelos conceituais relevantes de atividade intencional, a situação entra em debate e por fim toma-se uma ação na situação para produzir melhoria.

Ao considerar a metodologia SSM uma abordagem prática (Ensslin, 2002) - em função de sua perspectiva orientada para a ação - para a compreensão de questões complexas, e com a finalidade de aprendizado e ação, discorre-se neste artigo considerações de como a SSM pode contribuir para melhoria de processos já existentes nas Instituições de Educação Tecnológica, especificamente aqueles que envolvem o gerenciamento do conhecimento visando agregar valor aos Cursos Superiores de Tecnologia.

A exemplo de Soares (1998) que discute o uso da metodologia em um ambiente acadêmico, o presente artigo apresenta-se como um exercício reflexivo sob a possibilidade de aplicação em instituições de educação profissionalizante que buscam aperfeiçoar processos considerados estratégicos para as mesmas.

\section{Metodologia}

O principal objetivo desse artigo é o de contribuir para o aperfeiçoamento dos processos considerados estratégicos para as Instituições de Educação Tecnológica através da adequação da metodologia SSM ao contexto do ensino profissionalizante visto que trata-se de uma metodologia diagnóstica que deve ser usada antes de se escolher a ferramenta tecnológica adequada ao processo que se está analisando.

Em relação à abordagem do problema, a pesquisa pode ser classificada como bibliográfica e para a construção do artigo recorreu-se a referenciais teóricos, análise de documentos e coleta de informações. 


\section{Desenvolvimento}

\subsection{Gestão do Conhecimento nas Instituições de Educação Tecnológica}

\subsubsection{A Influência da Gestão do Conhecimento nas Organizações Intensivas em}

Conhecimento

O gerenciamento do conhecimento tem sido um dos principais parâmetros avaliados quando se pretende analisar quais os incrementos que determinada organização tem feito em prol de sua produtividade e desempenho. Ele é considerado um dos investimentos intangíveis mais relevantes juntamente com a geração e adoção de Tecnologias da Informação e Comunicação (TIC's) e a biotecnologia.

Segundo o Manual de Oslo (1997) as instituições que se baseiam em conhecimento devem incentivar processos interativos através "dos quais o conhecimento é criado e compartilhado dentro e entre organizações."

As organizações intensivas em conhecimento tem se proliferado alavancadas pela evolução da economia baseada em conhecimento tais como: empresas de consultoria, institutos de pesquisa, companhias de turismo, serviços bancários e as Instituições de Ensino Superior.

Estas têm reconhecido a necessidade de "ampliarem o uso de tecnologias intensivas em conhecimento para processos de produção e provisões de serviços." (Manual de Oslo, 1997) e de investirem em funcionários altamente qualificados.

Nessa perspectiva, as instituições de educação tecnológica têm um papel relevante dentro do mercado educativo brasileiro, pois os serviços oferecidos pelas mesmas vão desde a oferta de cursos e programas de formação inicial e continuada de trabalhadores, educação profissional técnica de nível médio, educação profissional tecnológica de graduação e pós-graduação, especialização técnica, seqüencial e ensino médio até serviços de assessoria e consultoria tecnológica.

Perante essa diversidade de atividades oferecidas e distintos níveis de qualificação de colaboradores, as instituições de educação tecnológica se caracterizam como organizações intensivas de conhecimento, pois possuem entre outros quesitos, uma "estrutura organizacional que conduz ao aprendizado e à exploração do conhecimento" (MANUAL DE OSLO, 1997).

Para Nadai (2007), como o conhecimento é o principal recurso dessas organizações, pode-se identificar as Instituições de Educação Tecnológica como organizações intensivas de conhecimento a partir da intensidade do conhecimento gerado nas interações entre os diferentes envolvidos nos processos educativos. 
Nesse sentido, a gestão do conhecimento desempenha um papel primordial, haja vista a complexidade de gerenciar tal diversidade de ativos intangíveis que fazem parte deste ambiente.

Sobre as formas de gerenciamento deste conhecimento são conhecidos vários estudos a respeito e que apresentam aproximações entre a dinâmica dos serviços oferecidos pelas Instituições de Educação Tecnológica e os processos inerentes à gestão do conhecimento.

\subsection{O Papel da Gestão do Conhecimento nos Processos Educativos das Instituições de} Educação Tecnológica

Baseados no levantamento de Leite (2006) são enfocadas diferentes etapas que fazem parte dos processos que envolvem a gestão do conhecimento sob a perspectiva de estudiosos da área.

Especificamente no estudo realizado por Stollenwerk (2001), tem-se uma visão generalizada sobre os diversos processos inerentes à gestão do conhecimento. $\mathrm{O}$ autor relata que o entendimento desses modelos se faz necessário para que possamos avaliar "a aplicabilidade da GC a processos intensivos em conhecimento" como aqueles existentes no contexto da educação profissional tecnológica de graduação.

Elaborou-se uma análise comparativa baseada nas semelhanças e interrelações usando como referencial o modelo de Nonaka e Takeuchi (1997). Por fazerem parte do itinerário do ambiente educacional das instituições de educação tecnológica, fez-se uma analogia (Quadro 1) entre os processos educativos relacionados ao ensino superior das mesmas e os processos básicos da GC.

Quadro 1 - Analogia entre processos do ensino superior das IET e processos de GC

\begin{tabular}{|c|c|}
\hline PROCESSOS DE GC & $\begin{array}{l}\text { PROCESSOS/ATIVIDADES DO ENSINO } \\
\text { SUPERIOR TECNOLÓGICO }\end{array}$ \\
\hline $\begin{array}{l}\text { Identificação } \\
\text { Mapeamento do conhecimento. Levantamento } \\
\text { de informações e conhecimentos que sao criados } \\
\text { e usados no ambiente da organização. }\end{array}$ & $\begin{array}{l}\text { Programas de incentivo à criatividade e } \\
\text { desenvolvimento de idéias, reunião de comitês } \\
\text { setoriais, incubadoras de empresas. }\end{array}$ \\
\hline $\begin{array}{l}\text { Aquisição } \\
\text { Processo de aquisição de conhecimentos } \\
\text { necessários para criação e manutenção de } \\
\text { competências essenciais. }\end{array}$ & $\begin{array}{l}\text { Visitas de benchmarking, seminários, semanas } \\
\text { acadêmicas, aquisição de livros didáticos, } \\
\text { periódicos e outros objetos de aprendizagem, TV } \\
\text { institucional. }\end{array}$ \\
\hline $\begin{array}{l}\text { Validação } \\
\text { Atividades para avaliar a qualidade do } \\
\text { conhecimento. }\end{array}$ & $\begin{array}{l}\text { Comitês de avaliação de qualidade do produto, } \\
\text { sistemas informatizados de pesquisa de satisfação } \\
\text { dos clientes. }\end{array}$ \\
\hline $\begin{array}{l}\text { Organização/Armazenagem } \\
\text { Garante a armazenagem do conhecimento para }\end{array}$ & $\begin{array}{l}\text { Banco de recursos didáticos, sistema de } \\
\text { gerenciamento de rotinas do processo educacional. }\end{array}$ \\
\hline
\end{tabular}




\begin{tabular}{|l|l|}
\hline $\begin{array}{l}\text { efetivar sua recuperação rápida, fácil e correra } \\
\text { através de sistemas efetivos. }\end{array}$ & \\
\hline $\begin{array}{l}\text { Compartilhamento/Disseminação } \\
\text { Mecanismo de distribuição do conhecimento } \\
\text { com a maior capilaridade possível. }\end{array}$ & $\begin{array}{l}\text { Rede interna de computadores, publicação de } \\
\text { periódicos, participação em congressos, portal do } \\
\text { aluno, semanas acadêmicas, ensino à distância. }\end{array}$ \\
\hline $\begin{array}{l}\text { Criação } \\
\text { Relacionado a criação de novas habilidades, } \\
\text { produtos ou processos mais eficientes. }\end{array}$ & $\begin{array}{l}\text { Trabalhos de conclusão de curso, apoio a projetos } \\
\text { de pesquisa, incubadoras de base tecnológica, } \\
\text { estabelecimento de parcerias com outras } \\
\text { Instituições. }\end{array}$ \\
\hline
\end{tabular}

Fonte: Adaptado de Leite (2006)

\section{Metodologia SSM (Soft Systems Methodology)}

A metodologia SSM (Soft Systems Methodology) ou Metodologia dos Sistemas Flexíveis foi desenvolvida no período de 1969 a 1972, por Peter Checkland na Universidade de Lancaster com o objetivo de enfrentar problemas administrativos reais, aplicando-se idéias sistêmicas a situações reais e usando a experiência adquirida para modificar tais idéias e sua metodologia de utilização (SIMONSEN, 1994).

Rossoni (2007) cita que o pensamento de Vickers (1983) influenciou o desenvolvimento da metodologia com a criação do conceito de apreciação, que é um ato mental, avaliativo, no qual normas conflitantes e valores determinam quais são os fatos relevantes, enquanto que fatos percebidos ou considerados exigem atenção, porque são vistos como relevantes para certas normas e valores. Ainda cita o autor que Vickers (1983) criou o sistema apreciativo a partir da crítica à decisão clássica. Seus esforços são dirigidos para a compreensão do processo social que são a essência das relações humanas, e para a natureza do julgamento de valor humano, contribuindo para a definição de sistemas de atividades humanas (que são formados por ações das pessoas) usada por Checkland.

A metodologia do sistema soft pode ser aplicada em problemas não estruturados, na definição problemática de objetivos, em sistemas sociais (Mauad et al, 2007), bem como nas disciplinas de Biologia, Ecologia, Economia, Demografia, Gestão, Engenharia, dentre outras. A metodologia utiliza-se de uma abordagem holística, para resolver problemas, os quais não podem ser resolvidos pela abordagem tradicional reducionista, com o fluxo da lógica baseada em indagações.

Para Ensslin (2002) o objetivo geral da SSM pode ser formulado em termos de se constituir como uma metodologia para facilitar a ação. A autora cita que o modelo aprimorado e proposto em 1981 foi um marco para evolução da SSM, pois o processo seqüencial da versão de 1972 foi substituído pela versão contendo sete estágios em um processo de aprendizagem circular, onde as 
setas do modelo inicial que então apresentavam-se em linha reta, passam a ser substituídas por setas que mostram uma estrutura lógica de um mosaico de ações, sem a obrigatoriedade de seguir sequencialmente os estágios de 1 a 7 (CHECKLAND, 1981) (Figura 1), enfatizando suas características de apoio à aprendizagem e à reformulação de hipóteses.

Os facilitadores da SSM devem (Traduzido e adaptado de COUPRIE et al, 2007):

(1) explorar uma situação problemática não estruturada,

(2) expressá-la,

(3) construir definições sucintas de sistemas relevantes,

(4) elaborar modelos conceituais desses sistemas,

(5) comparar os modelos com a situação problemática expressada,

(6) reunir mudanças culturalmente possíveis e sistemicamente desejáveis,

(7) sugerir ações para transformação da situação problemática.

Figura 1: Estágios da Metodologia SSM (Soft Systems Methodology)

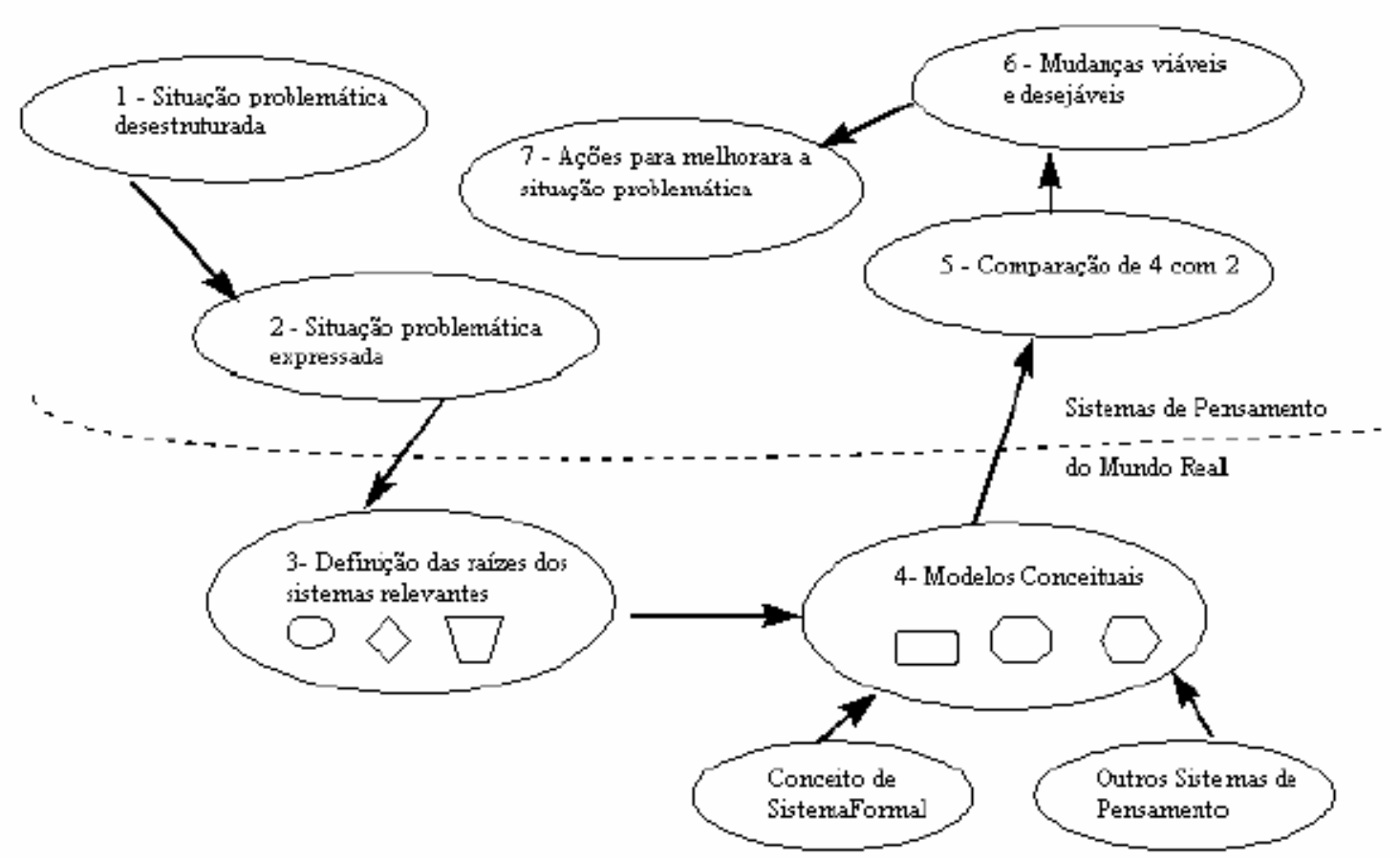

Fonte: Rossoni, 2007 (Traduzido e adaptado de Checkland, 1981)

Enfatiza-se que a SSM é uma metodologia, onde se destacam dois tipos de atividade; a que envolve o "mundo real" onde evidenciam-se as pessoas, as situações problemas e sua análise cultural, e a dos "sistemas mentais", onde uma análise lógica tenta envolver os participantes para a elaboração de modelos conceituais. 
Nos estágios 1 e 2 são desenvolvidos "retratos" da situação problema com a maior riqueza de detalhes possível, são trazidas informações fiéis da realidade, sem estrutura pré-concebida. Nestes estágios são expressas as situações para que os pontos relevantes sejam revelados.

No estágio 3 são identificados os sistemas relevantes para o problema ("definição de raízes dos sistemas relevantes" ou "raiz do problema"), os participantes são conduzidos do "mundo real" para os "modelos mentais". Após definidos detalhadamente, devem ser acompanhados com a identificação dos elementos $\boldsymbol{C A T W O E}$, nome atribuído por Checkland (1981) para o conjunto de elementos utilizado para que se defina no que consistem os sistemas (ENSSLIN, 2002). A sigla CATWOE foi transcrita a partir de Checkland e Scholes (1990)): C [Customers] clientes/beneficiários, $\boldsymbol{A}$ [Actors] atores/conduzem as atividades, $\boldsymbol{T}$ [Transformation process] processo de transformações/ entradas e saídas do sistema, $\boldsymbol{W}$ [Weltanschauung] visão de mundo/percepção, $\boldsymbol{O}$ [Owner] decisores/detentores do problema, $\boldsymbol{E}$ [Environment] ambiente/restrições externas.

No estágio 4 deve ser construído o modelo conceitual, a descrição dos meios necessários para que o sistema represente realmente a situação desejada.

O estágio 5 compara o modelo conceitual com a realidade descrita no estágio 2 . Os participantes da situação devem ser envolvidos no processo e as mudanças necessárias devem ser elencadas, base da discussão sobre as mudanças passíveis de serem implementadas (transformação da realidade), o que é feito no estágio 6, para que no estágio 7 sejam implementadas, levando-se em conta as ações julgadas relevantes para as mudanças desejadas.

É relevante citar que no estágio 5, o analista passa dos modelos mentais e inicia um debate considerando mudanças desejáveis ou viáveis, e que neste momento levanta discussões para comparar os modelos construídos no estágio anterior com a situação problema. Checkland (1981) descreve a comparação como um confronto entre "o que" com "como". O autor afirma que há quatro formas de realizar o "confronto":
a) discussão informal;
b) questionamento formal;
c) descrição de cenários baseado em modelos operacionais, reconstruído na seqüência dos eventos no passado;
d) "construir" o modelo "mundo real" da mesma forma que o "modelo conceitual" e comparar.


A partir de então passa-se a implementar as mudanças improvisadas na situação problema (estágios 6 e 7), lembrando que a metodologia SSM não é linear, interações devem ser realizadas e que no debate (estágio 5) se pode retornar a análise inicial e as definições "raiz".

Checkland (1981) afirma que a aplicação da metodologia permite mudanças em três categorias:

- Estruturas organizacionais;

- Atividades/procedimentos;

- Atitudes.

Checkland e Scholes (1990) revisaram e atualizaram a metodologia SSM (Figura 2), que passou a ser vista como uma opção para abordagem geral de problemas e não mais como uma metodologia de sete estágios para resolver problemas.

O problema inicial (situação do mundo real) é vista como produto de uma determinada história, a qual deve receber atenção especial, pois fornecerá a base para a compreensão da situação e na utilização da SSM (Figura 2) acompanhará dois distintos, mas integrados "fluxos" de análise.

Couprie et al (2007) citam que no "fluxo baseado em lógica" os envolvidos (atores) desenvolvem uma série de descrições dos chamados sistemas relevantes, modelados e, comparados com a situação percebida, que servem para estruturar as discussões sobre mudança.

Descreve também que no "fluxo de análise cultural" existem três principais exames do problema inicial (situação do mundo real):

- Análise da intervenção (análise e reflexão sobre a aplicação da SSM para o problema situação, criação das "paisagens ricas- Análise do sistema social (papéis, normas e valores da situação problema);

- Análise do sistema político (responder a perguntas relacionadas com a distribuição do poder no problema inicial).

Para o autor, com base na comparação do fluxo baseado na lógica e análises, é possível definir entre mudanças sistemicamente desejáveis e culturalmente possíveis de se implementar. A fase final está relacionada a realizar as mudanças propostas. 


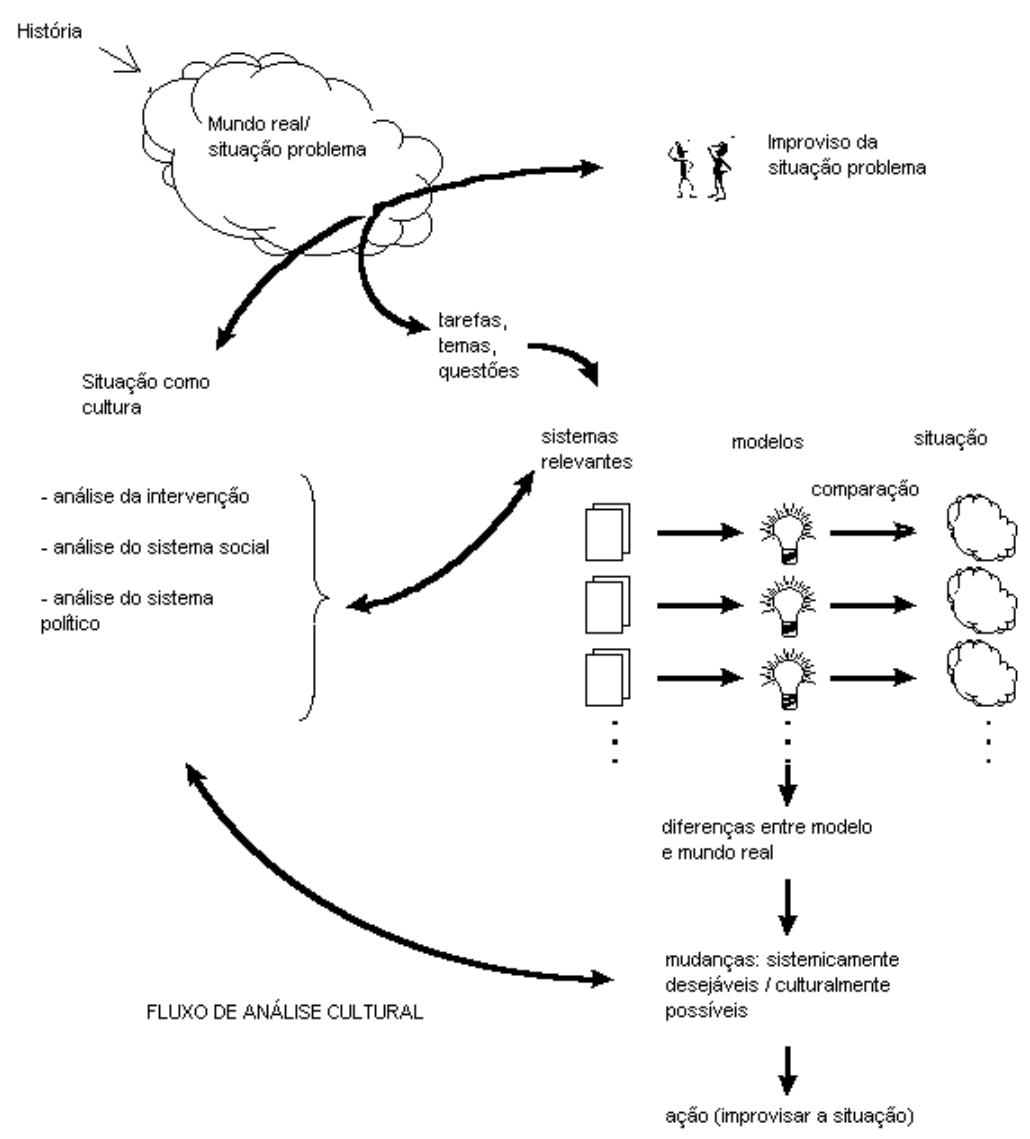

LÓGICA BASEADA NA ANÁLISE DE FLUXO

Fonte: Traduzido e adaptado de Couprie (2007)

\section{Avaliação do Processo Ensino-Aprendizagem nos Cursos Superiores de Tecnologia}

A partir de estudos referentes aos processos de gestão do conhecimento realizado na análise de literatura especializada é possível sugerir em qual(ais) dele(s) a SSM traria importantes contribuições ao ser adotada nos diversos processos educacionais que norteiam os cursos superiores de tecnologia.

\subsection{O Processo de Avaliação e sua Contribuição à Construção do Conhecimento}

Avaliar, assim como planejar, é uma ação comum no dia-a-dia de todos nós. Muito do que planejamos para as nossas vidas foi pensado a partir de nossas próprias avaliações. Tanto o planejamento como a avaliação são processos articulados e indissociados da ação educativa e da concepção do conhecimento. Toda avaliação tem uma intenção, que é a de garantir que aprendizagem se efetive, porém por muito tempo, e ainda hoje, avaliar significa apenas qualificar, 
medir, selecionar, separando os alunos que alcançam os resultados esperados daqueles que não conseguem, confirmando seu caráter classificatório e excludente. Geralmente este tipo de avaliação é feita apenas ao final do processo, ao final de um semestre, por exemplo, no intuito de aprovar ou reprovar um aluno, desconsiderando todo o processo de construção do conhecimento. O foco da avaliação deve estar no acompanhamento da trajetória de cada um, na apropriação das competências e dos objetivos previstos.

Autores como Hoffmann (1994) definem avaliação como "meio de acompanhamento do processo ensino-aprendizagem" enquanto outros como Vasconcellos (1995), fazem uma distinção entre avaliação e nota. Enquanto a avaliação seria um processo mais abrangente, implicando reflexão crítica sobre a prática e possibilitando uma tomada de decisão sobre o que fazer para melhorar o processo de aprendizagem, a nota seria somente uma exigência do sistema educacional. Limitar o ato de avaliar ao de atribuir uma nota leva a um desvio bastante comum.

Sob esta ótica, avalia-se para acompanhar o processo de aprendizagem, repensar a prática educativa, na busca de novas maneiras de desenvolver o trabalho, procurando sempre adequá-lo às necessidades dos alunos. A excelência do processo avaliativo está em ser útil na informação que oferece, viável na realização de sua trajetória, ética em seus propósitos e conseqüências e precisa na elaboração de seus instrumentos e no tratamento de seus dados. Requer o entendimento da reflexão constante sobre as dificuldades dos envolvidos e na oferta de novas possibilidades de aprendizagem.

Considerando que os Cursos Superiores de Tecnologia devem dar conta das exigências de um mercado de trabalho cada vez mais dinâmico e competitivo, e tendo em vista ainda o curto tempo para a integralização das disciplinas, fica evidente a necessidade de se esclarecer ao aluno como se dará a construção de seu conhecimento durante o período que estiver na Instituição de Educação Tecnológica, à luz dos objetivos especificados em cada umas das disciplinas e de um perfil profissional de conclusão pensado e elaborado para dar conta das demandas de determinada área de atuação.

\subsection{Adequacao da Metodologia SSM para Melhorias no Processo de Avaliação em Cursos Superiores de Tecnologia}

A metodologia SSM tem como principal objetivo identificar e estruturar situações problemáticas e com dificuldades de definição, visando de forma encadeada, relacionar o mundo real e o mundo do pensamento sistêmico, para se chegar às ações que promovam a solução de um problema. É dirigida a objetivos, uma vez que considera o sistema real e os meios para se alcançar um sistema desejável. Para tanto, prevê avaliação, controle, monitoração e feedback. 
A partir dos sete estágios propostos por Checkland, sugere-se a estruturação da situação problemática da seguinte forma:

(1) O que de fato o aluno precisa saber? O que ele precisa saber fazer? O que ele precisa saber ser? O que ele precisa saber para agir? (Levando em conta a situação problemática mal definida).

(2) Para explicitar a situação problemática, deve-se confrontar os objetivos e ementas de cada uma das disciplinas previstas na matriz curricular com o perfil profissional de conclusão do curso, no sentido de apontar que capacidades ou habilidades devem ser desenvolvidas pelo aluno em cada um dos conteúdos ministrados para dar conta da formação pretendida. A partir do mapeamento dessas capacidades criam-se os indicadores de desempenho, que nada mais são do que pistas ou indícios que ajudam o professor a perceber se aquelas capacidades elencadas são atingidas. A partir deste ponto, uma série de instrumentos ou atividades podem ser definidos como meio de verificação destes indicadores.

(3) Em seguida constroem-se definições sucintas de sistemas relevantes através de um conjunto de sistemas definidos por Checkland como $\boldsymbol{C A T W O E}$. Devem ser identificados os clientes (C) do processo, neste caso, os alunos da Instituição de Educação Tecnológica; os atores (A) do processo: corpo docente, discente, técnico-pedagógico e administrativo; o objeto de transformação (T), o entendimento que envolve o processo avaliativo do aluno; visão de mundo (W), reconhecendo a complexidade do processo ensino-aprendizagem focado em um sistema avaliativo inclusivo e formativo; o proprietário $(\mathrm{O})$ neste caso a Instituição de Educação Tecnológica; e, por fim, o levantamento de restrições (E) que podem impactar na solução do problema, neste caso, questões relacionadas a mudança cultural, já que a sociedade em que se vive é fruto de um sistema avaliativo classificatório, muitas vezes punitivo e excludente; além do tempo disponível para a implantação das idéias geradas.

(4) A partir daí pode-se elaborar um modelo conceitual do sistema pretendido, que sintetiza os caminhos para a construção da nova proposta de avaliação.

(5) Comparando-se o modelo criado com a situação problemática expressada, podem ser levantadas as seguintes questões: Em que consiste um sistema de avaliação ideal? Qual é a sua função? Quais são os pressupostos básicos norteadores das ações educativas e das práticas avaliativas?

No estágio seguinte (6) devem ser feitas reuniões com todos os docentes no intuito de fundamentá-los teoricamente sobre a evolução do processo avaliativo, na perspectiva de vários autores, sensibilizando-os para a importância da mudança. Neste momento, várias ações podem ser sugeridas para a transformação da situação problemática (7) a partir do seguinte questionamento: 
Como objetivar e tornar claro o processo avaliativo na instituição, de forma que o aluno perceba sua evolução no que tange a construção do seu conhecimento?

A partir deste momento, é possível acompanhar e a validar os critérios e indicadores de desempenho ao longo do período de estudo, com a participação de todos os envolvidos no processo. Além disso, vislumbra-se ainda com o novo sistema avaliativo implantado, mostrar ao aluno que ele também é responsável por seu desempenho e pela construção de seu conhecimento, que é desafiante, transformador e lhe fornece autonomia.

\section{Considerações Finais}

Entende-se que o principal resultado da aplicação da metodologia SSM é o aprendizado. Apresenta características democráticas e interativas, propiciando o diálogo, a geração e o confronto de idéias entre os envolvidos; é flexível, por se adequar a diversos cenários, nos seus mais variados contextos; é focada em objetivos, o que lhe garante foco; favorece o pensamento sistêmico; e, organiza uma a discussão de problemas, bem como suas possíveis soluções, embora não produza respostas e/ou resultados finalizados.

Patching (2007) destaca que a aplicação da metodologia SSM:

1) encoraja o analista a considerar questões e temas problemáticos, ao invés de problemas específicos;

2) promove melhor entendimento acerca de fraquezas organizacionais, e, pode revelar o porquê de problemas;

3) não impõem soluções tecnológicas ou modismos; e

4) exige a participação de envolvidos na situação problemática, evitando-se a formulação de políticas alheias à realidade da organização.

Finalmente, cabe citar que a utilização da metodologia SSM por pautar-se em uma visão integrada, permite minimizar a distância entre os níveis organizacionais a fim de aperfeiçoar o processo de gestão. Porém, torna-se limitada ao ser aplicada em ambientes intransigentes e autocráticos, que evitam confrontos de idéias ou que apresentam alta rotatividade de colaboradores (sem "times" bem estruturados não há como implementar as soluções oriundas da mesma). Outrossim, limita-se também por não propor respostas finais para a solução do problema, visto que questionamentos nunca terminam. 


\begin{abstract}
This article describes the Soft Systems Methodology (SSM) procedures utilization, as well as its applicability in Technological Education context. It analyzes the evaluative process in Graduation Technology Courses and how it can contribute to knowledge construction. It propose, therefore, this methodology application in a Technological Education Institution trying to solve the evaluative process problems, considering the subjectivity of this results based on concepts instead of grades like the traditional system; time necessary to integrate disciplines, and the learning process inherent to this mode, built upon the theoretical knowledge (know), on the practical skill development (know how to do) and the development of attitudes in human activity specific spheres (know how to be). The methodology adoption in educational processes that involves knowledge management in of Technological Education Institutions contributes to aggregates value to Graduation Technology Courses in addition minimizes the distance between the different organizational levels in order to improve the management process.
\end{abstract}

Keywords: Soft Systems Methodology, Technological Education, Knowledge Management, Technology Graduation Courses.

\title{
Referências
}

APPOLINARIO, F. Dicionário de Metodologia Científica: um guia para a produção de conhecimento científico. São Paulo: Atlas, 2004.

CHECKLAND, P. B. Systems Thinking, Systems Practice. Chichester: John Wiley, 1981.

CHECKLAND, P. B \& SCHOLES, J. Soft Systems Methodology in Action. Chichester: John Wiley, 1990.

COUPRIE, D. et al. Soft Systems Metodology. Calgari: Departament of Computer Science, University of Calgary, 1997. Disponível em: <http://www.cpsc.ucalgary.ca/cpsc_research/areas/evolutionary.> Acesso em: 10 nov. 2007.

ENSSLIN, S. R. A incorporacao da perspectiva sistêmico-sinergética na metodologia MCDA-Construtivista: uma ilustração de implementação. 2002. 478 f. Tese (Doutorado) - Curso de Programa de Pós-graduação em Engenharia de Produção, UFSC, Florianópolis.

HOFFMANN, J. Avaliação mediadora: uma prática em construção - da pré-escola à universidade. 4. ed. Porto Alegre: Educação e Realidade, 1994

LEITE, F.C.L. Gestão do conhecimento científico no contexto acadêmico: proposta de um modelo conceitual. 2006. 240p. Dissertação (Mestrado em Ciência da Informação)- Universidade de Brasília, Brasília, 2006.

LIMA FILHO, D. L. Formação de tecnólogos: lições da experiência, tendências atuais e perspectivas. Boletim Técnico do Senac, Rio de Janeiro, v. 25, n. 3, p. 41-53, set. /dez., 1999. Disponível em <www.senac.br>. Acesso em: 18 set. 2007

Manual de Oslo: diretrizes para coleta e interpretação de dados sobre inovação, $3^{\text {a }}$ ed, OCDE e Eurostat, Finep, 1997.

MANFREDINHO, N.P.S. Construção do conhecimento na perspectiva da educação tecnológica. 2000. $108 \mathrm{f}$. Dissertação (Mestrado em Engenharia da Produção)-Universidade Federal de Santa Catarina, Florianópolis, 2000.

MARTINELLI, D. P. Negociação Empresarial: Enfoque Sistêmico e Visão Estratégica. Manole. Barueri (SP). 2002.

MAUAD, T. M ET AL. (2003) Análise comparativa entre distritos industriais: uma aplicação do enfoque sistêmico para avaliar projetos de desenvolvimento local. Management in Iberoamerican Countries: Current Trends and Future Prospects Third International Conference. SP: FGV/EAUSP. Disponível em: $<\mathrm{http}: / /$ www.fgvsp.br/iberoamerican/Papers/0112_Artigo\%20IAM_final\%20formatado.pdf > Acesso em: 10 dez. 2007.

NADAI, F. C. O conhecimento como recurso estratégico: caracterizando uma organização intensiva em conhecimento (OIC). Disponível em: <http://www.ead.fea.usp.br>. Acesso em: 16 dez. 2007.

NONAKA,I; TAKEUCHI, H. Criação do conhecimento na empresa. 4.ed. Rio de Janeiro:Campus, 1997. 
PATCHING, D. Seeking out the issues: how soft systems methodology was employed to advise a social services department on the use of information technology. OR Insight, v. 5, n. 1. 1992, pp. 9-14. In: BELLINI, C. G. P.; RECH, I.; BORENSTEIN, D. Soft Systems Methodology: Uma aplicação no "Pão dos Pobres" de Porto Alegre. Disponível em: $\quad<$ http://www.rae.com.br/eletronica/index.cfm?FuseAction=Artigo\&ID=1790\&Secao=> INFORMAÇÃO RAEeletrônica, v. 3, n. 1, Art. 3, jan./jun. 2004. Acesso em: 15 nov. 2007.

cross ${ }^{\text {ref }}$

ROSSONI, L. Modelagem e simulação SOFT em estratégia. In: II Seminário de Gestão e Negócios. UniFAE, 2005. Disponível em: < http://www.inf.pucrs.br/ gilberto/tgs/mapas\%20cognitivos2.pdf> Acesso em: 10 nov. 2007.

SIMONSEN, J. Soft Systems Methodology. An Introduction Computer Science. Roskilde University. 1994.

SOARES, V.M.S. A soft systems methodology e a pesquisa-ação para projeto. In: SEMINÁRIO SOBRE METODOLOGIA DE PROJETOS DE EXTENSAO. Extensão universitária e Metodologia participativa. Anais. Rio de Janeiro: COPPE/UFRJ, 1998.

STOLLENWERK, M.F.L. Gestão do conhecimento: conceitos e modelos. In: TARAPANOFF, K. Inteligência organizacional e competitiva. Brasília: Editora Universidade de Brasília, 2001, 344 p.

VASCONCELOS, C. Construção do conhecimento. São Paulo: Vozes, 1995.

VICKERS, G. Human Systems are Different. London: Harper \& Row, 1983.

\section{Dados dos autores:}

Nome completo: Denize Demarche Minatti Ferreira

Filiação institucional: Universidade Federal de Santa Catarina (UFSC)

Departamento: Programa de Pós-Graduação em Engenharia e Gestão do Conhecimento (PPEGC)

Função ou cargo ocupado: Doutoranda

Endereço: EGC/ CTC/ Campus Universitário - Universidade Federal de Santa Catarina (UFSC),

Bairro Trindade, Florianópolis, Santa Catarina, Brasil, CEP 88040-970

Telefones para contato: (48) 9622-8363

e-mail:dminatti@terra.com.br

Nome completo: Arleide Rosa da Silva

Filiação institucional: SENAI - Serviço Nacional de Aprendizagem Industrial/SC

Departamento: Núcleo Ambiental

Função ou cargo ocupado: Especialista em Gestão

Endereço completo para correspondência: Rua Alfredo Morsch, 465 - Bairro: Velha/ Blumenau/SC

Telefones para contato: (47) 33281013 (resid.) - 99636155 - 33219652 e 33219674 (comercial)

e-mail: arleide@sc.senai.br

Nome completo: Teresa Cristina Santos Rebello

Filiação institucional: Universidade Estadual de Santa Catarina - UDESC 
Departamento: Moda

Função ou cargo ocupado: Professor Substituto

Endereço completo para correspondência: Universidade Estadual de Santa Catarina - UDESC, Av.

Madre Benvenutta, 1907, Itacorubi, Florianópolis, SC, Brasil, CEP: 88.035-001

Telefones para contato: (48) 8408-8218

e-mail: tete.rebello@gmail.com

Nome completo: Neri dos Santos

Filiação institucional: Universidade Federal de Santa Catarina (UFSC)

Departamento: Programa de Pós-Graduação em Engenharia e Gestão do Conhecimento (PPEGC)

Função ou cargo ocupado: Chefe do Departamento de Engenharia do Conhecimento

Endereço completo para correspondência: Endereço: EGC/ CTC/ Campus Universitário -

Universidade Federal de Santa Catarina (UFSC), Bairro Trindade, Florianópolis, Santa Catarina,

Brasil, CEP 88040-970. Caixa Postal No 5213

Telefones para contato: (48) 3721-7117

e-mail:neri@egc.ufsc.br

Recebido para publicação em: 28/09/2009

Aceito para publicação em: 09/11/2009 